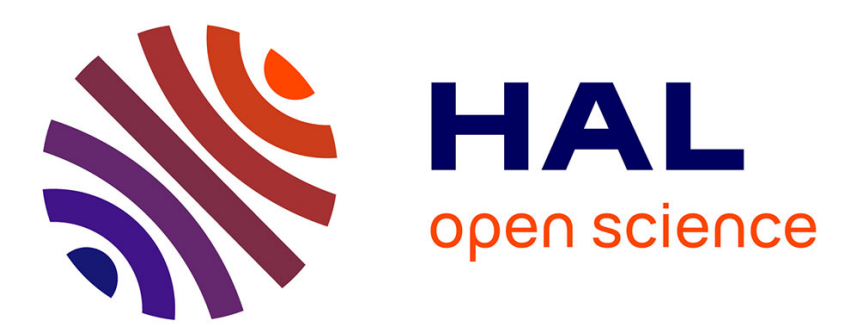

\title{
2D Numerical Modeling of a Bulk HTS Magnetization Based on H Formulation Coupled with Electrical Circuit
}

Jakub Kapek, Kévin Berger, Michael Rudolf Koblischka, Frédéric Trillaud, Jean Lévêque

\section{- To cite this version:}

Jakub Kapek, Kévin Berger, Michael Rudolf Koblischka, Frédéric Trillaud, Jean Lévêque. 2D Numerical Modeling of a Bulk HTS Magnetization Based on H Formulation Coupled with Electrical Circuit. IEEE Transactions on Applied Superconductivity, 2019, 29 (5), pp.1-5. 10.1109/TASC.2019.2897331 . hal-01987943v3

\section{HAL Id: hal-01987943 \\ https://hal.science/hal-01987943v3}

Submitted on 25 Feb 2019

HAL is a multi-disciplinary open access archive for the deposit and dissemination of scientific research documents, whether they are published or not. The documents may come from teaching and research institutions in France or abroad, or from public or private research centers.
L'archive ouverte pluridisciplinaire HAL, est destinée au dépôt et à la diffusion de documents scientifiques de niveau recherche, publiés ou non, émanant des établissements d'enseignement et de recherche français ou étrangers, des laboratoires publics ou privés. 


\title{
2D Numerical Modeling of a Bulk HTS Magnetization Based on $\mathbf{H}$ Formulation Coupled with Electrical Circuit
}

\author{
Jakub Kapek, Kévin Berger, Michael Rudolf Koblischka, Frederic Trillaud and Jean Lévêque
}

\begin{abstract}
Bulk High Temperature Superconductors (HTS) can be magnetized and act as permanent magnet much stronger than conventional ones as NdFeB. The design of the inductor is a key point to perform the desired magnetization of the HTS bulk. In this paper, we focus on modeling a Pulsed Field Magnetization (PFM) process of an HTS bulk using a coil powered with a magnetizer. The built model is a $2 \mathrm{D}$ axisymmetric problem, based on the $H$ formulation and coupled with electrical equations though the magnetic flux seen by the magnetizing coil. The calculation of this magnetic flux in the $\mathbf{H}$ formulation is not trivial and was validated using magnetic vector potential formulation on a coil in the air. Assuming different operating conditions, the bulk HTS is then modeled using four different properties corresponding to air, perfect diamagnetic, copper and HTS. It was shown that simulating a PFM process could lead to different value of peak current and applied magnetic field to the bulk HTS, depending on the critical current density of the bulk, for example. These variations are in the range of the air and diamagnetic cases. Therefore, the proposed method should be used in order to predict a realistic trapped magnetic field in the HTS bulk by taking into account its reaction seen by the coil during the PFM process.
\end{abstract}

Index Terms-Bulk conductors, coils, cuprates, eddy currents, electromagnetic transient analysis, $H$ formulation, magnetization processes.

\section{INTRODUCTION}

$\mathbf{T}$ HE YBCO material belongs to High Temperature Superconductors (HTS) and it was quickly used in the laboratory due to its good properties. For this superconducting material, the critical temperature $T_{\mathrm{c}}$ is $92 \mathrm{~K}$, while the temperature of liquid nitrogen is $77 \mathrm{~K}$, thus it allows an easy use in many applications. The HTS bulk can be magnetized and act as a permanent magnet with a remanent magnetic field 10 times stronger than conventional ones as $\mathrm{NdFeB}$ material. One of the most common method of magnetization is to cool down the HTS and place it inside a magnetizing coil during a Pulse Field Magnetization (PFM) process. The discharge of the magnetizer

The authors would like to acknowledge the AFFCCS and the IAEM doctoral school of the University of Lorraine for their financial support.

J. Kapek, K. Berger, and J. Lévêque are with the University of Lorraine, GREEN, Research Group in Electrical Engineering of Nancy - EA 4366, Faculté des Sciences et Technologies, BP 70239, 54506 Vandoeuvre-lès-Nancy Cedex, France (Corresponding author: K. Berger, email: kevin.berger@univlorraine.fr).

M. R. Koblischka was with the Experimental Physics, Saarland University, 66041 Saarbrücken, Germany. He is now with the Department of Materials Science and Engineering, Shibaura Institute of Technology, Tokyo 135-8548, Japan (email: m.koblischka@mx.uni-saarland.de).

F. Trillaud is with the Instituto de Ingeniera, Universidad Nacional Autnoma de Mexico, D.F., 04510 Mexico (email: ftrillaudp@ii.unam.mx) causes a flowing current through the coil, which produces a high external magnetic field, thus the bulk can trap magnetic fields, later marked as $B_{\text {trapp }}$. The process of magnetization requires designing a copper coil able to produce a magnetic field around a few Tesla. To achieve a the high value of applied magnetic field, a capacitor bank as impulse magnetizer system with an appropriate stored energy can be used. Moreover, in order to estimate the discharge of current properly, one key issue is to take into consideration the change of the magnetic flux seen by the coil during the magnetization process. This variation can be represented as varying inductance in the electrical equations. Thus, Maxwell's equations have to be coupled through the total magnetic flux with the electrical equations of the magnetizer.

Nowadays, numerical methods have become a more common approach to analyze the behavior of HTS. Finite Element Method (FEM) allows calculating electromagnetic quantities as the current density $J$ and the magnetic field $B$ induced in the HTS [1]. However, solving eddy current problems with rapidly changing magnetic fields can be a challenge even with a commercial software. There are several ways to simulate superconducting material; however, the most convenient formulations for HTS bulks have been selected here: H [2]-[4] and $\mathbf{A}-V$ [5].

In this paper, we focus on the modeling of a PFM process of an HTS bulk using a coil coupled with an electrical circuit. Most of the publications do not consider the coupling between the coil and the magnetizer in $\mathbf{H}$ formulation [6]-[8], what in our opinion can lead to discrepancies in the estimation of the trapped magnetic field in HTS bulk. Our model is based on the $\mathbf{H}$ formulation implemented using the PDE module and the global equation module in COMSOL Multiphysics 5.2a. For comparison and validation of the proposed method, an equivalent problem based on an A- $V$ formulation has been simulated with a definition of magnetic flux based on the magnetic vector potential. One of the aims of this paper is to see if some changes occurred on the applied magnetic field waveform when the HTS behaves as different materials, and to see the limit of validity of the assumptions usually made. The next sections describe the coupled numerical model, its validation and some numerical results during the PFM of a HTS bulk.

\section{Numerical Modeling of HTS}

There are few methods for magnetizing HTS [9]. In the following work, the PFM is used. This process consists of 


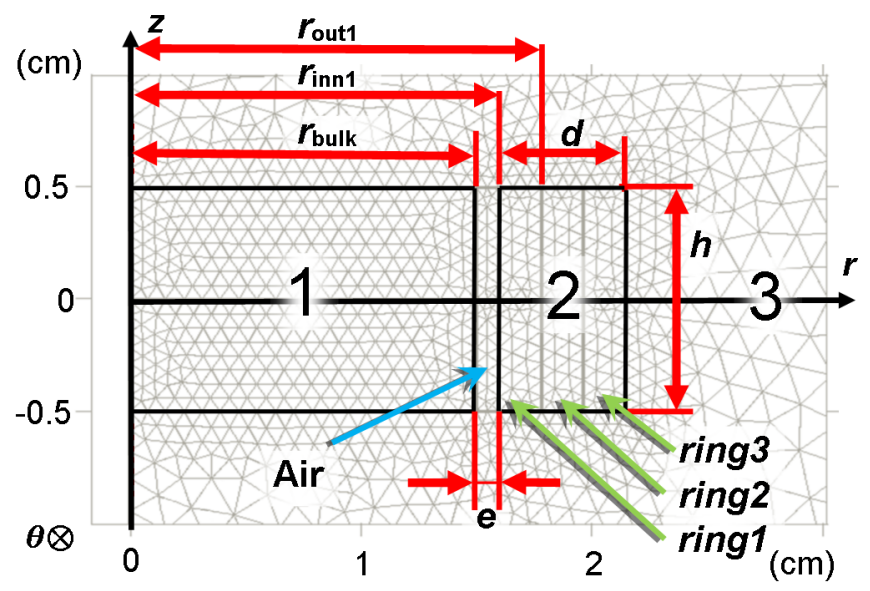

Fig. 1. Investigated model with the coil defined as three rings, where domain 1 is the bulk, 2 the coil and 3 represents the air

suddenly discharging the capacitor connected with the coil. The current flowing from the magnetizer to the coil must be big enough to produce the desired magnetic field of a few Tesla. The magnetization of HTS bulks may be divided into three physical stages detailed hereinafter. At low field, right at the beginning of the magnetization process, no volume current flows into the superconductor. Hence, it expels all the external magnetic field and behaves as a diamagnetic material, characterized by a magnetic susceptibility $\chi=-1$ $\left(\mu_{r}=1+\chi=0\right)$. At some point, the HTS is assumed to be in the mixed state with $\mu_{r}=1$, the induced currents flow into the superconductor due to the electric field that arises from the variations of the magnetic field, i.e. Lenz's law. In this state, for HTS materials, a nonlinear power law [10] is usually used to describe the $E(J)$ relationship, refer as

$$
E(J)=E_{\mathrm{c}}\left(\frac{J}{J_{\mathrm{c}}}\right)^{n}
$$

with $E_{\mathrm{c}}=1 \mu \mathrm{V} / \mathrm{cm}$. At liquid nitrogen temperature, the parameters $J_{\mathrm{c}}=100 \mathrm{~A} / \mathrm{mm}^{2}$ and $n=21$ correspond to reasonable values found in the literature [11]. When the bulk becomes fully penetrated by current and if its magnetization $M=B / \mu_{0}-H$ is weak compared to the huge applied external magnetic field, it can be assumed that the superconductor behaves like air with also $\mu_{r}=1$. At the end of the PFM, the external magnetic field disappears and the superconductor acts like a conductor with no losses and an internally flowing current density, generally close to $\pm J_{\mathrm{c}}$.

\section{Modeling}

\section{A. Specifications of the model}

The geometry of the 2D axisymmetric problem is shown in Fig. 1. The bulk is a cylinder with a radius $r_{\text {bulk }}=15 \mathrm{~mm}$ and a height $h=10 \mathrm{~mm}$. The coil, made of copper consists of $N=22$ turns in a total width $d=5.5 \mathrm{~mm}$ and has the same height as the bulk. The space between the bulk and the coil is $1 \mathrm{~mm}$ and marked as $e$. The cross-section of the coil is subdivided in three regions, further called 'rings', in order to improve the calculation of magnetic flux. The process of

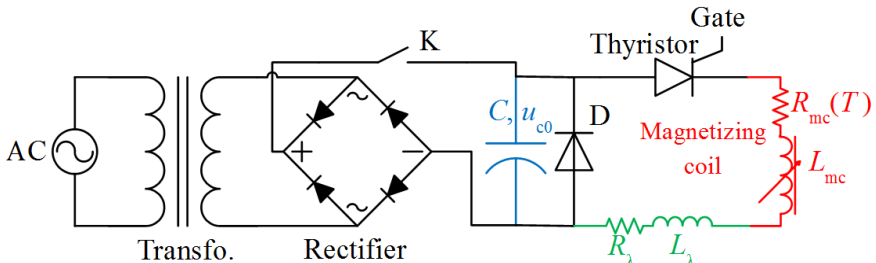

Fig. 2. Schematic of the magnetizer connected with the coil represented by $R_{\mathrm{mc}}, L_{\mathrm{mc}}$. The elements $R_{\lambda}, L_{\lambda}$ represent the parasitic elements in the circuit.

magnetization needs to produce a sufficient external magnetic field greater than the penetration field $B_{\mathrm{PB}}$ [11], thus the magnetizing coil, also called inductor, must be coupled with a suitable magnetizer. The available capacitor bank is capable of accumulating $10 \mathrm{~kJ}$ of energy with a repetitive maximum current of $25 \mathrm{kA}$. The capacity of the magnetizer is $5 \mathrm{mF}$ with the possibility of charging up to $2 \mathrm{kV}$. The electrical circuit of the magnetizer is shown in Fig. 2. The magnetizing coil represented by $R_{\mathrm{mc}}$ and $L_{\mathrm{mc}}$ is connected in series with the magnetizer. Due to the HTS placed inside the coil, the inductance seen by the magnetizer varies over time.

\section{B. PDE methodology}

The power law (1) can be modeled as a nonlinear resistivity,

$$
\rho(J)=\frac{E_{\mathrm{c}}}{J_{\mathrm{c}}}\left|\frac{J}{J_{\mathrm{c}}}\right|^{n-1}
$$

A common approach is to link the equation of the resistivity (2) with the $\mathbf{H}$ formulation [1]- [3]. Nowadays, this coupling seems to be the most suitable method for our problem [1], [4], [12], which can also provide good convergence and an accurate current distribution inside the bulk. These benefits classify the $\mathbf{H}$ formulation as a convenient approach for solving electrical equations during the magnetization process of HTS. The $\mathbf{H}$ formulation can be implemented in the PDE module, according to Faraday‘s law:

$$
\frac{\partial\left(\mu_{0} \mu_{r} \mathbf{H}\right)}{\partial t}+\nabla \times \mathbf{E}=0
$$

where $\mu_{r}$ is the relative permeability of the material. For the 2D axisymmetric model, the magnetic vector $\mathbf{H}$ has two variables $\left(H_{\mathrm{r}}, 0, H_{\mathrm{z}}\right)$ and the electric field $\mathbf{E}$ only one $\left(0, E_{\theta}, 0\right)$. To complete the formulation, two more terms must be defined: the curl product of magnetic field, which is Ampere's law written as

$$
J_{\theta}=\frac{\partial H_{\mathrm{r}}}{\partial z}-\frac{\partial H_{\mathrm{z}}}{\partial r}
$$

and the electric field defined as

$$
E_{\theta}=\rho J_{\theta}-\rho J_{\mathrm{a}}
$$

The additional term $J_{\mathrm{a}}$ represents an applied current in the coil. In FEM software, to overcome the difficulty of the eddy current in the coil, the resistivity of the coil can be increased until a uniform imposed current is obtained in the coil. This technique requires to include the proper resistance of the coil in the electrical equation. 


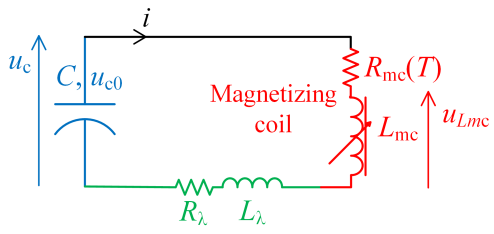

Fig. 3. Electrical circuit defined in COMSOL Multiphysics. The electrical model of the whole system is an RLC circuit with $L_{\mathrm{mc}}$, the variable inductance of the coil. is

The electrical equation for the RLC circuit shown in Fig. 3

$$
-u_{c}+\left(R_{\lambda}+R_{\mathrm{mc}}\right) i+L_{\lambda} \frac{\mathrm{d} i}{\mathrm{~d} t}+u_{L_{\mathrm{mc}}}=0
$$

with the current defined as

$$
i=-C \frac{\mathrm{d} u_{\mathrm{c}}}{\mathrm{d} t},
$$

where the term $u_{L_{\mathrm{mc}}}$ is the voltage drop across the designed coil. By using Faraday's law it can be shown that the changing of magnetic flux with respect to time is equal to the voltage drop across the element $L_{\mathrm{mc}}$

$$
u_{L_{\mathrm{mc}}}=+\frac{\mathrm{d} \Phi}{\mathrm{d} t}
$$

where $R_{\mathrm{mc}}$ is the resistance of the coil kept constant during the PFM process. To be more realistic, additional parasitic elements with the script $\lambda$ have been included. These elements represent the cables, their connections and the internal resistance of the capacitors. These are determined when the magnetizer is short-circuited, i.e. without coil $R_{\lambda}=4 \mathrm{~m} \Omega$ and $L_{\lambda}=4 \mu \mathrm{H}$ in our case. The electrical equation (6) corresponds to the magnetizer without diode and thyristor. The diode is assumed as a perfect element and when the capacitor voltage reaches zero, the diode starts to conduct, and the voltage is kept to zero until the end of the PFM process.

In order to take into account the reaction of the HTS bulk during the PFM process, an expression of the magnetic flux seen by the coil must be defined. Due to the fact that the coil is not a discrete punctual element, the definition of the magnetic flux seen by the coil is not trivial in the FEM software. For simplification, a calculation of the magnetic flux in the coil by dividing it into three sub-domains (rings) is proposed, see Fig. 1. The magnetic flux seen by the coil is then calculated by taking an average over the width and the height of each ring. For that, the magnetic flux is integrated over the height of the ring for the inner and outer surfaces and the mean value is calculated by

$$
\langle\phi\rangle_{1}=\frac{1}{2 h}\left(\int_{h} \int_{S_{\text {inn1 }}} \mathbf{B} \cdot \mathrm{d} \mathbf{S}_{\text {inn1 }} \mathrm{d} h+\iint_{h} \int_{S_{\text {out } 1}} \mathbf{B} \cdot \mathrm{d} \mathbf{S}_{\text {out } 1} \mathrm{~d} h\right)
$$

with $\mathrm{d} \mathbf{S}_{\text {inn1 }}=r_{\text {inn } 1} \mathrm{~d} r \mathrm{~d} \theta \hat{\mathbf{u}}_{z}$ and $\mathrm{d} \mathbf{S}_{\text {out } 1}=r_{\text {out } 1} \mathrm{~d} r \mathrm{~d} \theta \hat{\mathbf{u}}_{z}$. For the whole coil, the average magnetic flux is then:

$$
\Phi(\mathbf{B})=N \cdot\left(\langle\phi\rangle_{1}+\langle\phi\rangle_{2}+\langle\phi\rangle_{3}\right)
$$

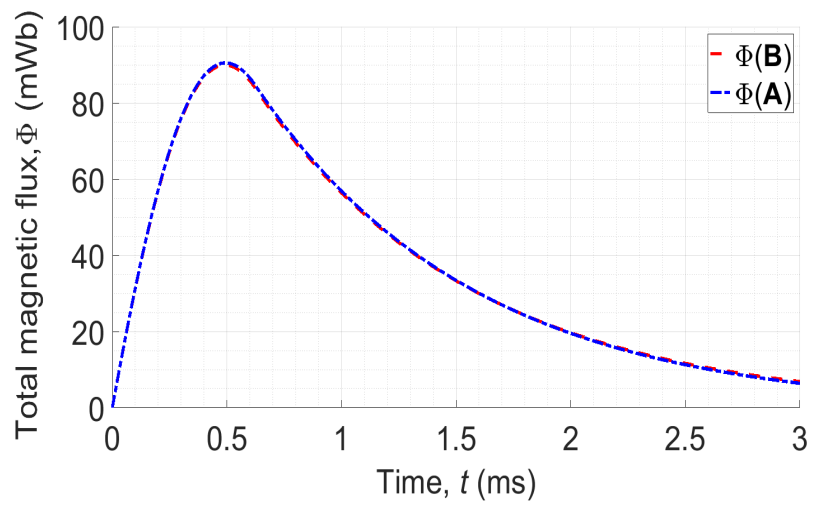

Fig. 4. Total magnetic flux for a model based on the $\mathbf{H}$ formulation with the coil defined as three rings. For this case, the coil was only surrounded by air.

\section{Existing modules in COMSOL Multiphysics}

The COMSOL program contains built-in module based on the A- $V$ formulation. The equation for this $\mathbf{A}$ formulation can be written as

$$
\sigma \frac{\partial \mathbf{A}}{\partial t}-\nabla \times \frac{1}{\mu_{0} \mu_{r}} \nabla \times \mathbf{A}=0
$$

The A formulation requires modeling the behavior of the HTS with a nonlinear conductivity as

$$
\sigma(E)=\frac{J_{\mathrm{c}}}{E_{\mathrm{c}}}\left|\frac{E}{E_{\mathrm{c}}}\right|^{\frac{1}{n}-1}
$$

The function $\sigma(E)$ is the reciprocal function of $\rho(J)$ (2), but its use leads to an ill conditioned matrix and also to a very difficult convergence for the FEM solver. This usually leads to a very noisy current distribution in the HTS [13]. The same model governed by the $\mathbf{A}$ formulation can be used to validate the $\mathbf{H}$ formulation coupled with the electrical equation but only for a coil surrounded by air.

The magnetic flux seen by the coil based on the magnetic vector potential can be written as

$$
\Phi(\mathbf{A})=N \cdot\left\langle\oint_{\partial S_{\text {coil }}} \mathbf{A} \cdot \mathrm{d} \mathbf{l}\right\rangle_{S_{\text {coil }}}
$$

with $\mathrm{d} \mathbf{l}=r \mathrm{~d} \theta \hat{\mathbf{u}}_{\theta}$. This corresponds to the average value of the magnetic vector potential in the whole domain of the coil. In the $\mathbf{A}-V$ formulation, the coupling with the electrical circuit has been directly implemented using electrical circuit interface in COMSOL Multiphysics.

\section{RESUlts AND Discussion}

To validate our model, we first focused on the calculation of the magnetic flux seen by the coil, when the bulk region is considered as air, because the A- $V$ formulation is not relevant for eddy currents problem in HTS. A discharge of the RLC circuit is made, assuming a perfect diode and an initial capacitor charge of $400 \mathrm{~V}$. This value is enough to fully magnetize the HTS sample within our setup since it can produce a magnetic field well above twice the full penetration field $B_{\mathrm{PB}}=1.14 \mathrm{~T}$ in our case. 
TABLE I

VALUES OF RESISTIVITY AND RELATIVE PERMEABILITY ASSIGNED TO THE BULK IN THE H FORMULATION.

\begin{tabular}{lcc}
\hline \hline Symbol & $\rho[\Omega \cdot \mathrm{m}]$ & $\mu_{r}$ \\
\hline Air & 1 & 1 \\
Diamagnetic & 1 & $1 \mathrm{e}-3$ \\
Copper & $1.67 \mathrm{e}-8$ & 1 \\
HTS & $E(J)$ law $(1)$ & 1 \\
\hline \hline
\end{tabular}

Fig. 4 shows the total magnetic flux seen by the coil using two different methods. The calculation of magnetic flux with (13) is assumed to be the reference and a comparison with the magnetic flux based on (10) is made. The calculated magnetic fluxes are fairly the same. Therefore, (10) can be later used to calculate the magnetic flux seen by the coil in the $\mathbf{H}$ formulation.

As mentioned in the introduction, the bulk HTS can behave as different materials and it has been modeled using four different properties corresponding to air, perfect diamagnetic, copper and HTS. Table I summarizes these properties used in the bulk region. All the simulations are made using the $\mathbf{H}$ formulation coupled with electrical equation (10).

Fig. 5 shows the time evolution of the current flowing through the coil during the PFM for bulk properties in Table I. When the bulk is modeled by air, there is no current inside the bulk region and it is equivalent to decouple the electrical equation to the $\mathbf{H}$ formulation and to use a constant inductance value of $L_{\lambda}$. It corresponds to the lowest value of peak current of $4.4 \mathrm{kA}$ and a time constant of $0.5 \mathrm{~ms}$. It can be mentioned here that in [6], the authors proposed an analytic expression of the inductance of a magnetizing coil in its environment, including an iron core. Taking into account that the bulk region is a perfect diamagnetic material leads to reach the other limit of the PFM process. Of course, this case corresponds to a current sheet and a strong reaction of the bulk seen by the coil. This reaction tends to decrease the inductance value $L_{\lambda}$ significantly, which results in a larger peak current of $5.4 \mathrm{kA}$ and a time constant of $0.35 \mathrm{~ms}$. Surprisingly, from the point of view of the coil and the waveform of the current, modeling the bulk region with the power law (1) and $J_{\mathrm{c}}=100 \mathrm{~A} / \mathrm{mm}^{2}$ corresponds to almost the same case than air. This result can be explained since the applied field is largely greater that the penetration field $B_{\mathrm{PB}}$ [11]. Finally, modeling the bulk region with copper leads to a different waveform of the current with a peak current of $4.6 \mathrm{kA}$ and a time constant of $0.38 \mathrm{~ms}$, between the air and diamagnetic cases.

In order to confirm that the air and diamagnetic cases are asymptotic limits for the current waveforms, the same PFM has been applied to the HTS bulk modeled using (1) but changing the critical density value for 1 to $10^{4} \mathrm{~A} / \mathrm{mm}^{2}$. Fig. 6 shows the evolution of the current waveform for four cases and as expected, if $J_{\mathrm{c}}$ is very high, the current in the coil is the same that the one corresponding to a perfect diamagnetic conductor and if $J_{\mathrm{c}}$ is very low, the current behaves like with the air case. If the penetration field $B_{\mathrm{PB}}$ and the applied magnetic field are in the same order of magnitude, then the current waveform is different and included within the range of

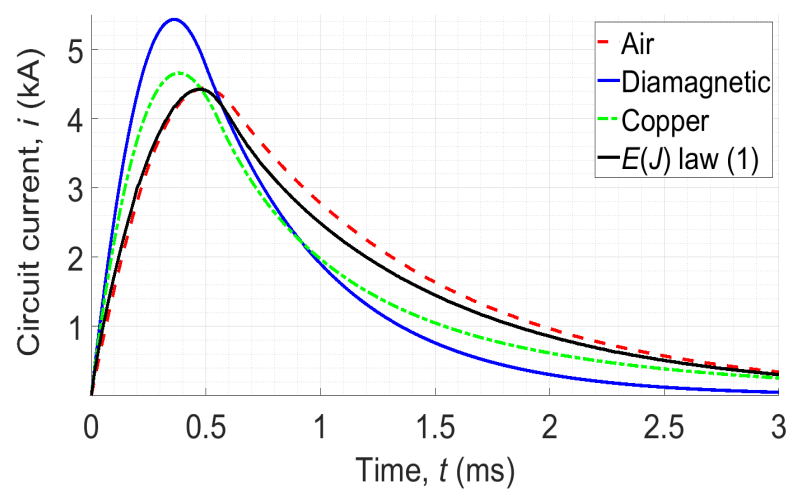

Fig. 5. Current flowing through the coil during the process of magnetization for bulk defined as air, perfect diamagnetic material, copper and using $E(J)$ law.

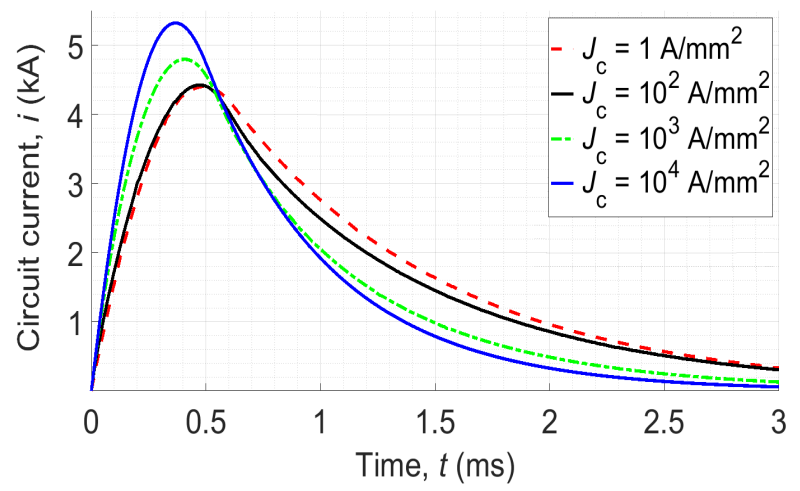

Fig. 6. Influence of the increase of the critical current of the HTS on the current flowing through the coil during the PFM process.

these two asymptotic behaviors. Neglecting the reaction of the bulk HTS on the magnetizing coil during PFM process can lead to an under-estimation of the peak current and applied magnetic field up to $20 \%$. This conclusion is reached using an inductor without magnetic material, but it has also been verified with a nonlinear magnetic material below the HTS bulk.

The magnetic flux density along the $r$ axis at $z=0$ is plotted in Fig. 7. Three moments during PFM are presented: (a) at the beginning of the magnetization $t=0.05 \mathrm{~ms}$, (b) at the maximum value of the external magnetic field $t=0.5 \mathrm{~ms}$ and (c) at the end of the magnetization $t=15 \mathrm{~s}$. The trapped magnetic field $B_{\text {trapp }}$ at the center of the bulk is $0.65 \mathrm{~T}$ at the end of the PFM process. For the same number of Degrees of Freedom (DoF), the computing time of the $\mathbf{H}$ formulation coupled with the electrical equations is about $10 \%$ more than the uncoupled one, on a Intel ${ }^{\circledR}$ Xeon $^{\circledR}$ CPU E5-2630 v4 @ $2.2 \mathrm{GHz}$.

\section{CONCLUSION}

This paper provides a new method for modeling an HTS bulk during PFM. The model based on $\mathbf{H}$ formulation coupled with the electrical equation and taking into account a power law for the HTS has been successfully simulated using COMSOL Multiphysics 5.2a. It was shown that simulating a PFM 


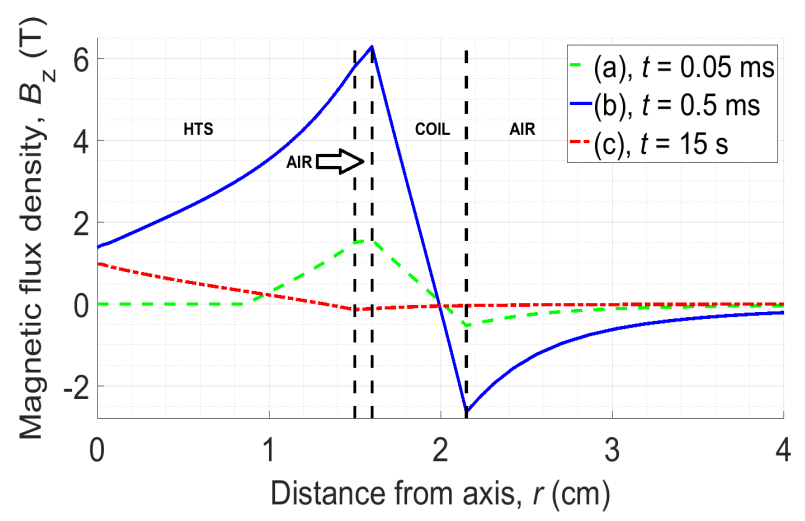

Fig. 7. Magnetic flux density during the PFM of the HTS along $r$ axis at $z=0$ and three moments of simulation. For $t=0.05 \mathrm{~ms}$ magnetic flux density start penetrate into bulk, at $t=0.5 \mathrm{~ms}$ the maximum external magnetic field appears. At $t=15 \mathrm{~s}$, the trapped magnetic field at the end of the magnetization is shown, with $B_{\text {trapp }}=0.65 \mathrm{~T}$ at the center of the bulk.

process with a given capacitor voltage and known setup could lead to different value of peak current and applied magnetic field to the bulk HTS, depending on the critical current density of the bulk, for example. These variations are in the range of the air and diamagnetic cases. The inductance value calculated in such asymptotic cases from analytically expressions can be used to estimate the peak current and time constant. Therefore, the proposed method should be used in order to predict a realistic trapped magnetic field in the HTS bulk by taking into account its reaction seen by the coil during the PFM process.

\section{REFERENCES}

[1] K. Berger, G. Escamez, L. Quéval, A. Kameni, L. Alloui, B. Ramdane, F. Trillaud, L. Makong Hell, G. Meunier, P. J. Masson, and J. Lévêque, "Benchmark on the 3D Numerical Modeling of a Superconducting Bulk," in 21st International Conference on the Computation of Electromagnetic Fields (Compumag 2017), Daejeon, South Korea, Jun. 2017, p. (ID 110). [Online]. Available: https://hal.archives-ouvertes.fr/hal-01548728v4

[2] R. Brambilla, F. Grilli, and L. Martini, "Development of an edge-element model for ac loss computation of hightemperature superconductors," Superconductor Science and Technology, vol. 20, pp. 16-24, 2006.

[3] M. Zhang and T. Coombs, "3d modeling of high-tc superconductors by finite element software," Superconductor Science and Technology, vol. 25, p. 015009, 2011.

[4] J. Rhyner, "Magnetic properties and ac-losses of superconductors with power law currentvoltage characteristics," Physica C: Superconductivity, vol. 212, no. 3-4, pp. 292-300, 1993.

[5] S. Stavrev, F. Grilli, B. Dutoit, N. Nibbio, E. Vinot, I. Klutsch, G. Meunier, P. Tixador, Y. Yang, and E. Martinez, "Comparison of numerical methods for modeling of superconductors," IEEE Transactions on Magnetics, vol. 38, no. 2, pp. 849-852, 2002.

[6] M. Elbaa, K. Berger, B. Douine, M. Halit, E. H. Ailam, and S. E. Bentridi, "Analytical modeling of an inductor in a magnetic circuit for pulsed field magnetization of hts bulks," IEEE Transactions on Applied Superconductivity, vol. 28, no. 4, p. 8201306, June 2018.

[7] J. Arnaud, J. F. Fernandes, and P. J. C. Branco, "Modifying the pulsed-field-magnetization technique for hts bulks in electrical machines without magnetic field sensors," IEEE Transactions on Applied Superconductivity, vol. 28, no. 4, p. 6801604, 2018.

[8] Z. Huang, H. S. Ruiz, and T. Coombs, "Pulsed field magnetization strategies and the field poles composition in a bulk-type superconducting motor," Physica $C$ : $S u$ perconductivity and its Applications, vol. 534, pp. 73-81, 2017.

[9] M. D. Ainslie and H. Fujishiro, "Modelling of bulk superconductor magnetization," Superconductor Science and Technology, vol. 28, no. 5, p. 053002, 2015.

[10] Z. Hong, A. Campbell, and T. Coombs, "Numerical solution of critical state in superconductivity by finite element software," Superconductor Science and Technology, vol. 19, no. 12, p. 1246, 2006.

[11] B. Douine, K. Berger, F. Trillaud, M. Elbaa, and E. H. Ailam, "Determination of the complete penetration magnetic field of a hts pellet from the measurements of the magnetic field at its top-center surface," IEEE Transactions on Applied Superconductivity, vol. 28, no. 4, p. 8800104, 2018.

[12] M. D. Ainslie, H. Fujishiro, T. Ujiie, J. Zou, A. Dennis, Y. Shi, and D. A. Cardwell, "Modelling and comparison of trapped fields in (re) bco bulk superconductors for activation using pulsed field magnetization," Superconductor Science and Technology, vol. 27, no. 6, p. 065008, 2014.

[13] O. Bíró, "Edge element formulations of eddy current problems," Computer methods in applied mechanics and engineering, vol. 169, no. 3-4, pp. 391-405, 1999. 